\title{
Synthesis of a High-Coercivity FePt-Ag Nanocomposite Magnet via Block Copolymer-Templated Self-Assembly
}

\author{
Hiroaki Wakayama and Hirotaka Yonekura \\ Toyota Central R\&D Laboratories, Inc., 41-1 Yokomichi, Nagakute, Aichi 480-1192, Japan \\ Correspondence should be addressed to Hiroaki Wakayama; wakayama@mosk.tytlabs.co.jp
}

Received 30 June 2017; Revised 28 September 2017; Accepted 30 October 2017; Published 15 November 2017

Academic Editor: Jean M. Greneche

Copyright (C) 2017 Hiroaki Wakayama and Hirotaka Yonekura. This is an open access article distributed under the Creative Commons Attribution License, which permits unrestricted use, distribution, and reproduction in any medium, provided the original work is properly cited.

\begin{abstract}
Magnetic recording media are composed of magnetic thin films consisting of magnetically isolated crystallites. For practical use of magnetic particles as recording media, it will be necessary to realize high coercivity by fabricating nanocrystalline grains and forming grain boundaries with the nonmagnetic phase. In this study, a high-coercivity FePt-Ag nanocomposite magnet was synthesized by means of block copolymer-templated self-assembly. Precursors of Fe, Pt, and Ag were introduced into a polymer block, and the resulting material was oxidized and then reduced to form a nanocomposite consisting of FePt nanoparticles surrounded by a matrix of Ag. X-ray diffraction analysis revealed that the introduction of $\mathrm{Ag}$ did not significantly affect the crystalline ordering of the FePt. The addition of Ag increased the coercivity by 53\% (from 11.1 to $17.0 \mathrm{kOe}$ ). Our results suggest that the grain boundaries of the nonmagnetic $\mathrm{Ag}$ metal acted as pinning sites, disrupting magnetic coupling between individual FePt nanocrystallites and hindering domain wall motion at an external magnetic field.
\end{abstract}

\section{Introduction}

Along with recent improvements in the processing capability of computers, the capacity of the hard disk drive as an external storage device has also dramatically increased. Magnetic recording media are rapidly becoming popular not only for personal computers but also for consumer electronics such as video recorders, and demand for such media will likely increase as high-resolution digital television broadcasting becomes more common. In addition, as the IoT develops, further data densification will be required so that digital data can be recorded for longer periods of time and so that the portability of related equipment using magnetic recording media can be increased by means of miniaturization.

Magnetic recording media are composed of magnetic thin films consisting of magnetically isolated crystallites [16]. Increasing the recording density requires a reduction in the medium area for a single bit, which in turn necessitates a proportional decrease in the volume of the magnetic particles constituting the medium. However, decreasing the particle volume results in deterioration of resistance to thermal disturbance and in magnetization reversal due to thermal fluctuation, and consequently information cannot be recorded on the medium [7-9].

The thermal fluctuation problem can be minimized by using magnetic recording media with a high magnetic anisotropy $\left(K_{u}\right)[10,11]$. The ordered $\mathrm{Ll}_{0} \mathrm{FePt}$ phase has $K_{u}$ of at least 10 times that of the currently used CoCr alloy system [12], and this material is the most promising candidate for a high- $K_{u}$ medium. However, for practical use of $\mathrm{Ll}_{0}$ ordered alloys as recording media, it will be necessary to realize high coercivity by fabricating nanocrystalline grains, controlling (001) orientation, and forming grain boundaries with the nonmagnetic phase $[10,13]$.

Sputtering is the most commonly used method for fabricating FePt nanostructures [14-18], but this method, which requires high vacuum and high energy, is too expensive for industrial applications. Fabrication of FePt nanostructures by chemical synthesis is a promising alternative, but achieving high coercivity with chemical synthesis methods has been difficult [19-22]. The problem has been attributed to the difficulty of controlling nanocomposite structures.

In this study, we synthesized FePt-Ag nanoparticles by using self-assembled block copolymer (BCP) nanostructures 


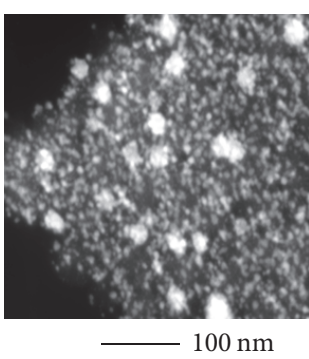

(a)

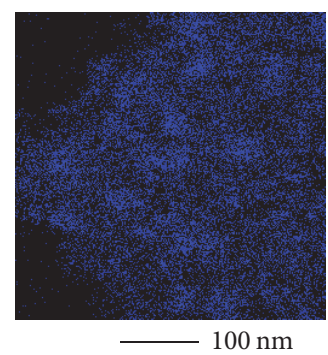

(b)

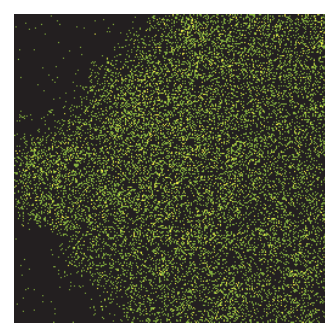

(c)

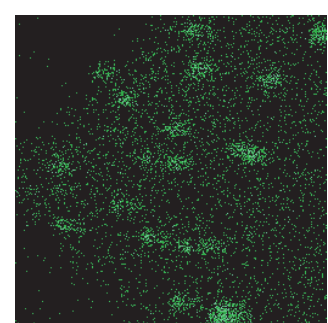

$-100 \mathrm{~nm}$

(d)

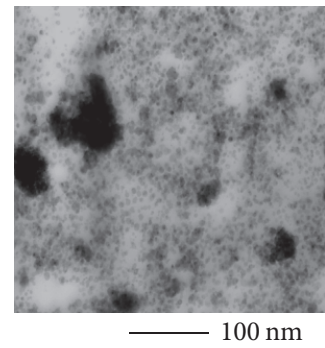

(e)

FIGURE 1: (a) Dark-field STEM image of sample synthesized with $\mathrm{Ag}$ and heat-treated at $180^{\circ} \mathrm{C}$, and (b)-(d) corresponding EDS mapping images for Pt, Fe, and Ag, respectively. (e) Bright-field STEM image of calcined sample synthesized with $\mathrm{Ag}$ heat-treated at $180^{\circ} \mathrm{C}$.

as templates to control the nanocomposite structure. Precursors of $\mathrm{Fe}, \mathrm{Pt}$, and $\mathrm{Ag}$ were introduced into one polymer block of the BCP, and then oxidation and reduction were used to remove the BCP template and crystallize the FePt nanocrystallites. We also investigated how the addition of nonmagnetic Ag affected the structure and magnetic properties of the FePt-Ag nanoparticles.

\section{Materials and Methods}

2.1. Sample Preparation. In a typical procedure, $0.13 \mathrm{~g}$ of tricarbonyl(cyclooctatetraene)iron (>96\%), $0.54 \mathrm{~g}$ of dimethyl(1,5-cyclooctadiene)platinum(II) (99\%), $0.57 \mathrm{~g}$ of tris(acetylacetonate)iron(III) (99\%), and $0.13 \mathrm{~g}$ of silver trifluoroacetate $(97 \%)$ were dissolved in a $0.5 \mathrm{wt} \%$ solution of polystyrene- $b$-poly(4-vinylpyridine) (PS-P4VP, $M_{n}{ }^{\mathrm{PS}}=22 \mathrm{~kg}$ $\mathrm{mol}^{-1}, M_{n}{ }^{\mathrm{P} 4 \mathrm{VP}}=22 \mathrm{~kg} \mathrm{~mol}^{-1}$, and polydispersity index = 1.09 ) in $45 \mathrm{~mL}$ of toluene (>99.5\%). After stirring for $3 \mathrm{~h}$ at room temperature, the solution was transferred to a Petri dish and calcined at $180^{\circ} \mathrm{C}$ for $6 \mathrm{~h}, 450^{\circ} \mathrm{C}$ for $6 \mathrm{~h}$, and $650^{\circ} \mathrm{C}$ for $1 \mathrm{~h}$ under a flow of $5 \% \mathrm{H}_{2}$ in Ar. Then the sample was oxidized at $550^{\circ} \mathrm{C}$ for $6 \mathrm{~h}$ in air and reduced at $450^{\circ} \mathrm{C}$ for $6 \mathrm{~h}$ under a flow of $5 \% \mathrm{H}_{2}$ in Ar. A reference sample was synthesized by means of the same protocol, but without silver trifluoroacetate.

\subsection{Characterization. Scanning transmission electron mi-} croscopy (STEM) images and energy-dispersive spectrometry (EDS) results were obtained with a JEOL JEM2010FEF(HR) transmission electron microscope operated at $200 \mathrm{keV}$. X-ray diffraction (XRD) patterns were collected with $\mathrm{Cu} \mathrm{K} \alpha$ radiation on a Rigaku RINT-TTR diffractometer operated at $40 \mathrm{kV}$ and $50 \mathrm{~mA}$. To measure magnetic properties, samples were saturated in a $100 \mathrm{kOe}$ pulsed magnetic field produced by a pulsed high-field magnetometer (TPM1-15, Toei Industry Co.), and the magnetic properties were measured at $300 \mathrm{~K}$ with a vibrating sample magnetometer (VSM-3S-15, Toei Industry Co.).

\section{Results and Discussion}

Dark-field and bright-field STEM images of a sample synthesized with $\mathrm{Ag}$ and heat-treated at $180^{\circ} \mathrm{C}$ showed the formation of spherical BCP-precursor composite mesophases with diameters of $40-100 \mathrm{~nm}$ (Figures 1(a) and 1(e)). The
TABLE 1: EDS spot analysis for C, Fe, Ag, and Pt of the synthesized sample presented in Figure 2.

\begin{tabular}{cccccc}
\hline & $\mathrm{C}($ at\%) & $\mathrm{Fe}($ at\%) & $\mathrm{Ag}(\mathrm{at} \%)$ & $\mathrm{Pt}(\mathrm{at} \%)$ & Total (at\%) \\
\hline 001 & 96.07 & 2.28 & 0.25 & 1.39 & 100.00 \\
002 & 75.36 & 8.16 & 6.62 & 9.86 & 100.00 \\
\hline
\end{tabular}

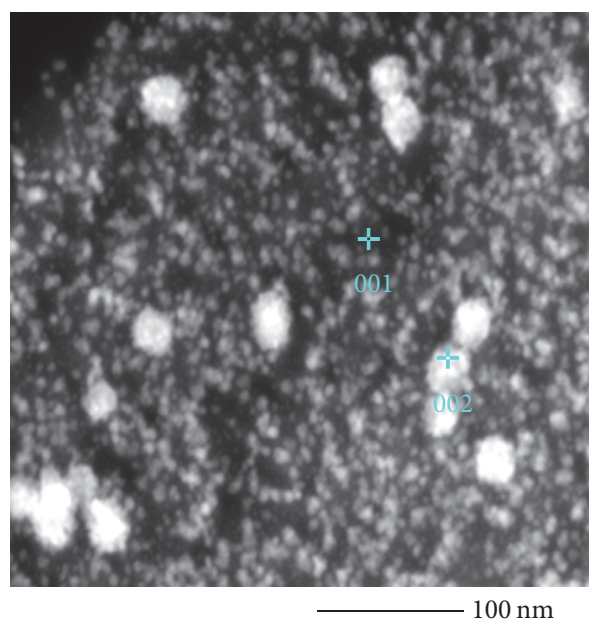

FIGURE 2: Dark-field STEM image of sample synthesized with Ag and heat-treated at $180^{\circ} \mathrm{C}$.

dark areas in the bright-field image correspond to moreelectron-dense areas, and the contrast observed in the image suggests that electron-dense elements were homogeneously distributed in the areas. The EDS mapping images in Figures 1(b)-1(d) show that the Fe, Pt, and Ag precursors were introduced into the P4VP blocks and that the Fe precursor was introduced into the PS matrix, which is consistent with previous reports $[23,24]$. Spherical mesophase formation was driven by the selective dissolution of the PS block in the toluene-based solvent [25], which resulted in dissolution of the Fe, Pt, and Ag precursors into the P4VP block.

EDS spot analysis of a sample synthesized with $\mathrm{Ag}$ and heat-treated at $180^{\circ} \mathrm{C}$ revealed that large portions of the $\mathrm{Fe}$, $\mathrm{Pt}$, and Ag precursors were introduced into the spheres, with smaller portions being introduced into the matrix (Figure 2 and Table 1). 


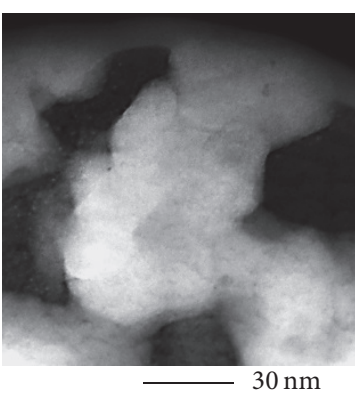

(a)

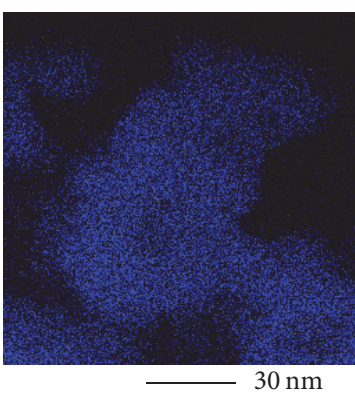

(b)

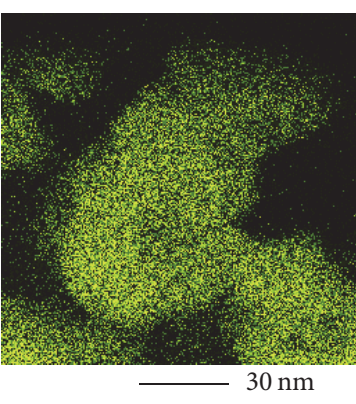

(c)

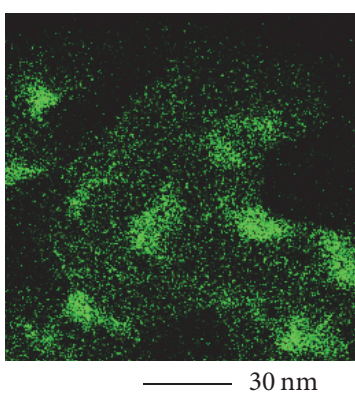

(d)

FIGURE 3: (a) Dark-field STEM image of oxidized and reduced sample synthesized with Ag, and (b)-(d) corresponding EDS mapping images for $\mathrm{Pt}, \mathrm{Fe}$, and $\mathrm{Ag}$, respectively.

SEM and EDS mapping images of oxidized and reduced sample synthesized with Ag clearly showed the distributions of $\mathrm{Fe}, \mathrm{Pt}$, and $\mathrm{Ag}$ after removal of the polymer templates. A dark-field STEM image shows condensed nanoparticles with diameters of 20-60 nm (Figure 3(a)), which corresponds almost exactly to the dimensions of the P4VP polymer blocks into which the $\mathrm{Fe}, \mathrm{Pt}$, and $\mathrm{Ag}$ precursors were introduced (Figure 1(a)). EDS mapping images show that these nanoparticles consisted of Fe and Pt and that they were surrounded by a matrix of Ag.

Peaks for an $\mathrm{Ll}_{0}$ tetragonal hard magnetic phase of $\mathrm{FePt}$ were observed in the XRD patterns of an oxidized and reduced sample synthesized without Ag (Figure 4(a)). The XRD profile of a sample synthesized with Ag confirmed the presence of two crystalline phases (Figure 4(b)): a $\mathrm{L}_{10}$ tetragonal hard magnetic phase of FePt containing $\mathrm{Ag}$ and a nonmagnetic phase of the Ag metal. The $\mathrm{L}_{10}$ tetragonal phase peaks were slightly shifted to a lower angle, indicating that the FePt was alloying with Ag. This result corroborates the EDS results; that is, a large portion of Ag was present at the grain boundary and a small portion of Ag was present within the FePt nanoparticles (Figure 3).

For bulk $\mathrm{L}_{10} \mathrm{FePt}, \boldsymbol{a}$ - and $c$-lattice parameters of 3.85 and $3.71 \AA$, respectively, have been reported [26]. Calculating the a- and c-lattice parameter values from the position of the (200) and (001) XRD peaks for the oxidized and reduced sample synthesized with Ag gives $a=3.87 \AA$ and $c=3.74 \AA$, which are a little larger than those reported for bulk $\mathrm{L}_{10}$ FePt. This difference in parameter values is likely due to expansion of the lattice through incorporation of $\mathrm{Ag}$ atoms and the altered $\mathrm{FePt}$ composition resulting from the reaction of $\mathrm{Fe}$ with a small amount of Ag.

These results indicate that the $\mathrm{Pt}, \mathrm{Fe}$, and Ag precursors introduced into the spherical polymer blocks (Figure 1) crystallized into $\mathrm{L}_{0} \mathrm{FePt}$ containing $\mathrm{Ag}$. In the formation of FePt nanoparticles, a large portion of the $\mathrm{Ag}$ within the spheres of the polymer template was extruded or diffused to the grain boundary. The spheres containing large amounts of Ag ultimately became FePt nanoparticles containing a small amount of Ag. We hypothesize that the diffusion distance of Ag was less than several tens of nanometers during the heat treatment up to $650^{\circ} \mathrm{C}$.

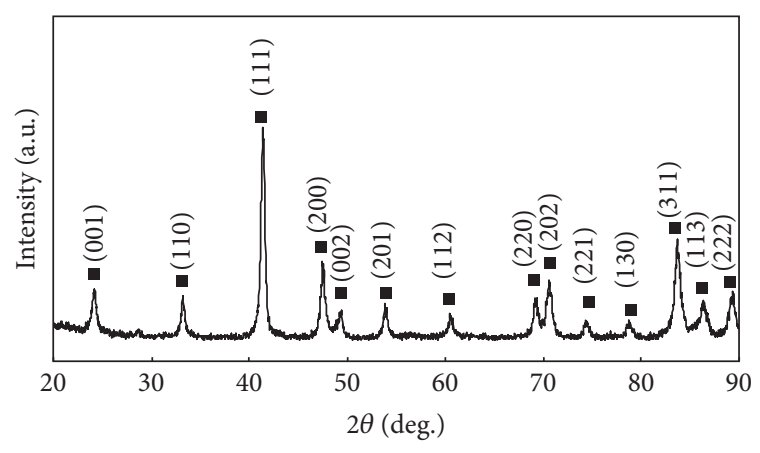

- $\mathrm{FePt}$

(a)

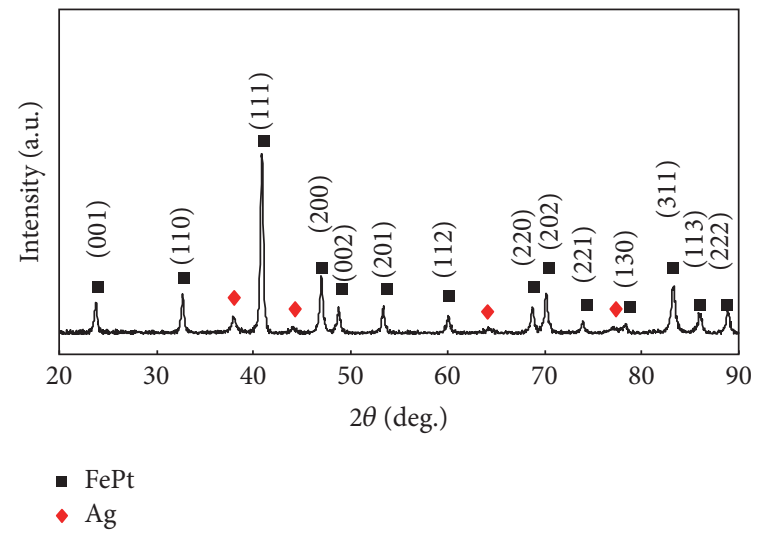

(b)

FIGURE 4: XRD patterns of oxidized and reduced sample synthesized (a) without Ag and (b) with Ag. FePt (black squares), Ag (red diamonds).

We evaluated the degree of chemical ordering in the samples by considering the uniaxial magnetocrystalline anisotropy of the $\mathrm{Ll}_{0} \mathrm{FePt}$ phase. The degree of chemical ordering is based on the crystalline ordering of the face-centered tetragonal structure and is described by the chemical order parameter $(S)$. This dimensionless parameter is given by the equation $S=0.85\left(I_{001} / I_{002}\right)^{1 / 2}$, where $I_{001}$ and $I_{002}$ are the 


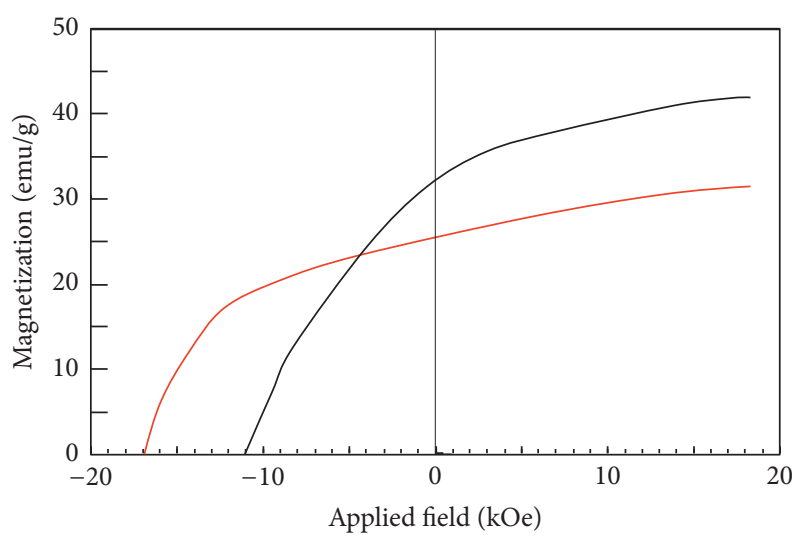

FIGURE 5: Magnetization curves of oxidized and reduced sample synthesized with $\mathrm{Ag}$ (red) and without Ag (black).

integrated intensities of the (001) and (002) peaks in the XRD spectrum, respectively [27]. The values of $S$ for calcined samples synthesized without $\mathrm{Ag}$ and with $\mathrm{Ag}$ were 0.944 and 0.955 , respectively. These values suggest that the introduction of Ag did not significantly affect the crystalline ordering of FePt. Ag diffusion promotes atomic diffusion, leading to a change in the chemical ordering of the $\mathrm{L}_{10}$ tetragonal phase after reduction. However, Ag diffusion had little effect on the promotion of this chemical ordering change. We hypothesize that the amount of $\mathrm{Ag}(13 \%)$ was insufficient to promote a change in the chemical ordering [19].

Magnetization curves of oxidized and reduced samples synthesized with $\mathrm{Ag}$ and without $\mathrm{Ag}$ (Figure 5) revealed that saturation magnetization of the sample synthesized with $\mathrm{Ag}(31.7 \mathrm{emu} / \mathrm{g})$ was $24 \%$ less than that of the sample synthesized without $\mathrm{Ag}(41.9 \mathrm{emu} / \mathrm{g})$. This result suggests that nonmagnetic species, possibly including Ag metal, lowered the saturation magnetization by decreasing the amount of magnetic substances in the sample.

The coercivity was increased by $53 \%$ (from 11.1 to $17.0 \mathrm{kOe}$ ) by the addition of Ag. The coercivity of the sample synthesized with Ag was much higher than the reported values for chemically synthesized FePt nanocomposites [19-22]. This suggests that the increase in the coercivity was caused by the addition of $\mathrm{Ag}$ to the FePt nanocrystals and that the grain boundaries of the nonmagnetic $\mathrm{Ag}$ metal acted as pinning sites, disrupting magnetic coupling between individual $\mathrm{FePt}$ nanocrystallites and hindering domain wall motion at an external magnetic field. This result is ascribed to the formation of well-controlled nanocomposite structures of hard magnetic FePt nanoparticles surrounded by nonmagnetic Ag metal, under the influence of templating by self-assembled $\mathrm{BCP}$ nanostructures.

\section{Conclusions}

Using self-assembled BCP templates, we synthesized a highcoercivity FePt-Ag nanocomposite magnet consisting of FePt nanoparticles surrounded by a matrix of Ag. XRD analysis revealed that the introduction of $\mathrm{Ag}$ did not significantly affect the crystalline ordering of the FePt. The coercivity increased by $53 \%$ (from 11.1 to $17.0 \mathrm{kOe}$ ) owing to the addition of Ag.

\section{Conflicts of Interest}

The authors declare no competing financial interests.

\section{Acknowledgments}

The authors greatly appreciate assistance from Ms. A. Mori with chemical synthesis and the transmission electron microscopy.

\section{References}

[1] G. Varvaro and F. Casoli, Ultra-High-density magnetic recording: Storage Materials and Media Designs, Pan Stanford, London, UK, 2016.

[2] E. Jafari-Khamse, M. Almasi-Kashi, A. Ramazani, and H. Almasi-Kashi, "The effect of the thickness ratio of magnetic layers on the microstructure and magnetic properties of (CoCrPt)97.5Nb2.5/Co75Cr13Pt12/Cr thin films," The European Physical Journal Plus, vol. 129, no. 12, article no. 276, 2014.

[3] B. Bhushan, Tribology and Mechanics of Magnetic Storage Devices, Springer New York, New York, NY, 1996.

[4] D. Weller and A. Moser, "Thermal effect limits in ultrahighdensity magnetic recording," IEEE Transactions on Magnetics, vol. 35, no. 6, pp. 4423-4439, 1999.

[5] D. Weller, A. Moser, L. Folks et al., "High $\mathrm{K}_{u}$ materials approach to 100 Gbits/in 2," IEEE Transactions on Magnetics, vol. 36, no. 1, pp. 10-15, 2000.

[6] D. Weller, G. Parker, O. Mosendz et al., "Review Article: FePt heat assisted magnetic recording media," Journal of Vacuum Science \& Technology B, vol. 34, no. 6, Article ID 060801, 2016.

[7] R. O. Topaloglu, More than Moore Technologies for Next Generation Computer Design, Springer, New York, NY, USA, 2015.

[8] B. K. Chatterjee, K. Bhattacharjee, A. Dey, C. K. Ghosh, and K. K. Chattopadhyay, "Influence of spherical assembly of copper ferrite nanoparticles on magnetic properties: Orientation of magnetic easy axis," Dalton Transactions, vol. 43, no. 21, pp. 7930-7944, 2014.

[9] W. F. Brown, "Thermal fluctuations of a single-domain particle," Physical Review A: Atomic, Molecular and Optical Physics, vol. 130, no. 5, pp. 1677-1686, 1963.

[10] K. Trohidou, Magnetic Nanoparticle Assemblies, Pan Stanford, 2014.

[11] R. Sbiaa, H. Meng, and S. N. Piramanayagam, "Materials with perpendicular magnetic anisotropy for magnetic random access memory," Physica Status Solidi - Rapid Research Letters, vol. 5, no. 12, pp. 413-419, 2011.

[12] O. A. Ivanov, L. V. Solina, V. A. Demshina, and L. M. Magat, "Determination of the anisotropy constant and saturation magnetization, and magnetic properties of powders of an ironplatinum alloy," The Physics of Metals and Metallography, vol. 35, no. 1, pp. 81-85, 1973.

[13] S. H. Charap, P.-L. Lu, and Y. He, "Thermal stability of recorded information at high densities," IEEE Transactions on Magnetics, vol. 33, no. 1, pp. 978-983, 1997. 
[14] S. Mi, R. Liu, Y. Li, J. Ye, Y. Xie, and Z. Chen, "Effect of sputter pressure on magnetotransport properties of FePt nanocomposites," Journal of Magnetism and Magnetic Materials, vol. 403, pp. $14-17,2016$.

[15] H. Wang, H. Zhao, T. Rahman et al., "Fabrication and characterization of fept exchange coupled composite and graded bit patterned media," IEEE Transactions on Magnetics, vol. 49, no. 2, pp. 707-712, 2013.

[16] Y.-J. Chiu, C.-Y. Shen, S.-R. Jian et al., "Nanoindentation study of FePt thin films deposited by radio frequency magnetron sputtering," Nanoscience and Nanotechnology Letters (NNL), vol. 8, no. 3, pp. 260-265, 2016.

[17] T. Hasegawa and K. Ito, "Structural Dependent FerromagneticNonmagnetic Phase Change in FePtRu Films," Advances in Materials Science and Engineering, vol. 2017, pp. 1-7, 2017.

[18] H. Pandey, A. Perumal, J. Wang, Y. K. Takahashi, and K. Hono, "Growth Mechanism of Columnar Grains in FePt-C Granular Films for HAMR Media Processed by Compositionally Graded Sputtering," IEEE Transactions on Magnetics, vol. 52, no. 7, 2016.

[19] H. Wang, H. Wang, P. Shang et al., "One-step synthesis of high-coercivity L 10-FePtAg nanoparticles: Effects of $\mathrm{Ag}$ on the morphology and chemical ordering of FePt nanoparticles," Chemistry of Materials, vol. 25, no. 12, pp. 2450-2454, 2013.

[20] Y. Yu, P. Mukherjee, Y. Tian, X.-Z. Li, J. E. Shield, and D. J. Sellmyer, "Direct chemical synthesis of L10-FePtAu nanoparticles with high coercivity," Nanoscale, vol. 6, no. 20, pp. 12050-12055, 2014.

[21] S. Sun, C. B. Murray, D. Weller, L. Folks, and A. Moser, "Monodisperse FePt nanoparticles and ferromagnetic FePt nanocrystal superlattices," Science, vol. 287, no. 5460, pp. 1989-1992, 2000.

[22] S. S. Kang, D. E. Nikles, and J. W. Harrell, "Synthesis, chemical ordering, and magnetic properties of self-assembled FePt-Ag nanoparticles," Journal of Applied Physics, vol. 93, no. 10, pp. 7178-7180, 2003.

[23] B.-H. Sohn, S.-I. Yoo, B.-W. Seo, S.-H. Yun, and S.-M. Park, "Nanopatterns by free-standing monolayer films of diblock copolymer micelles with in situ core - Corona inversion," Journal of the American Chemical Society, vol. 123, no. 50, pp. 12734-12735, 2001.

[24] A. Horechyy, N. E. Zafeiropoulos, B. Nandan et al., "Highly ordered arrays of magnetic nanoparticles prepared via block copolymer assembly," Journal of Materials Chemistry, vol. 20, no. 36, pp. 7734-7741, 2010.

[25] C. Burda, X. Chen, R. Narayanan, and M. A. El-Sayed, "Chemistry and properties of nanocrystals of different shapes," Chemical Reviews, vol. 105, no. 4, pp. 1025-1102, 2005.

[26] No. 00-043-1359, JCPDS International Center for Diffraction Data, 2016.

[27] B. E. Warren, X-Ray Diffraction, Dover Books on Physics, Dover Books on Physics, New York, NY, USA, 1990. 

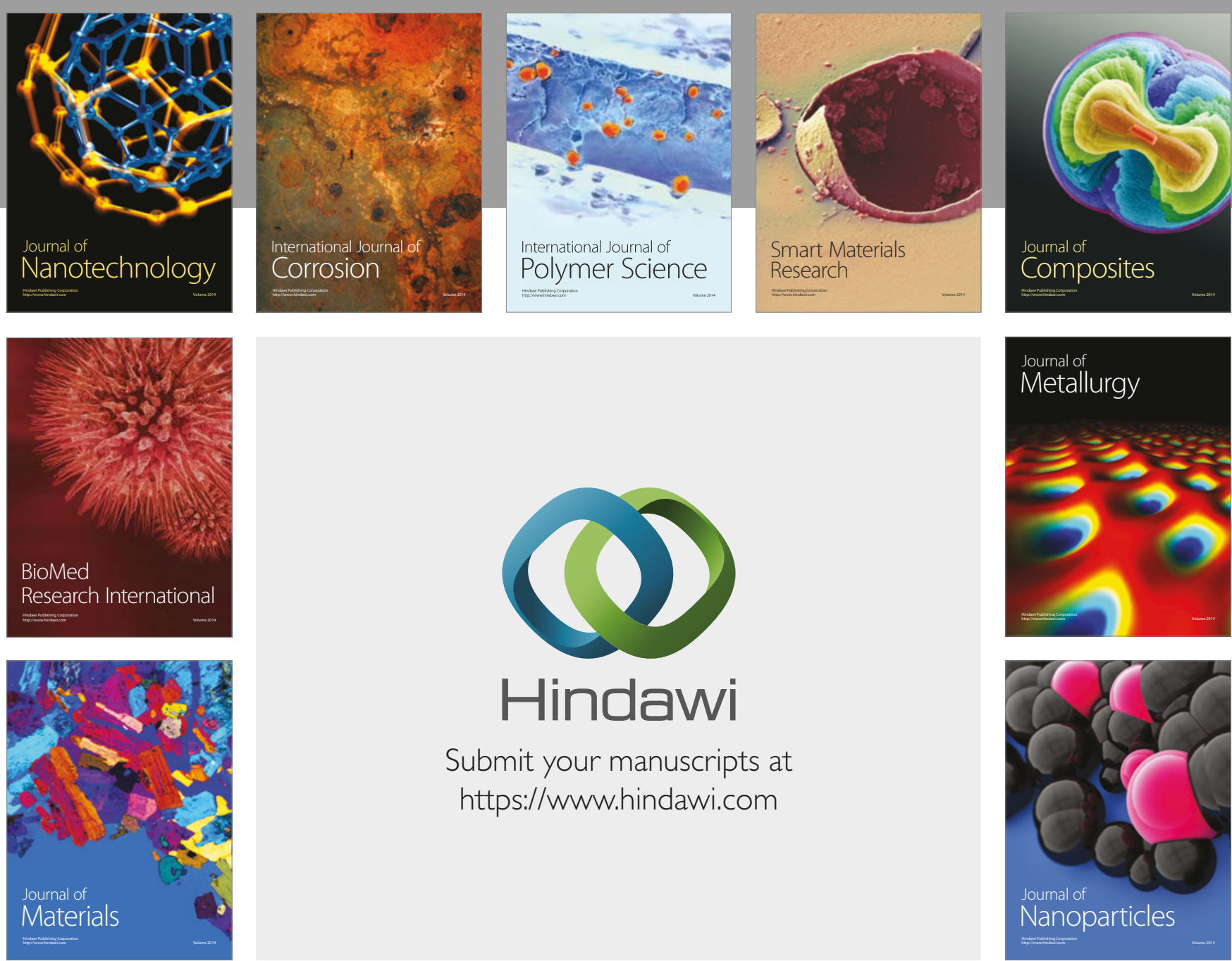

\section{Hindawi}

Submit your manuscripts at

https://www.hindawi.com
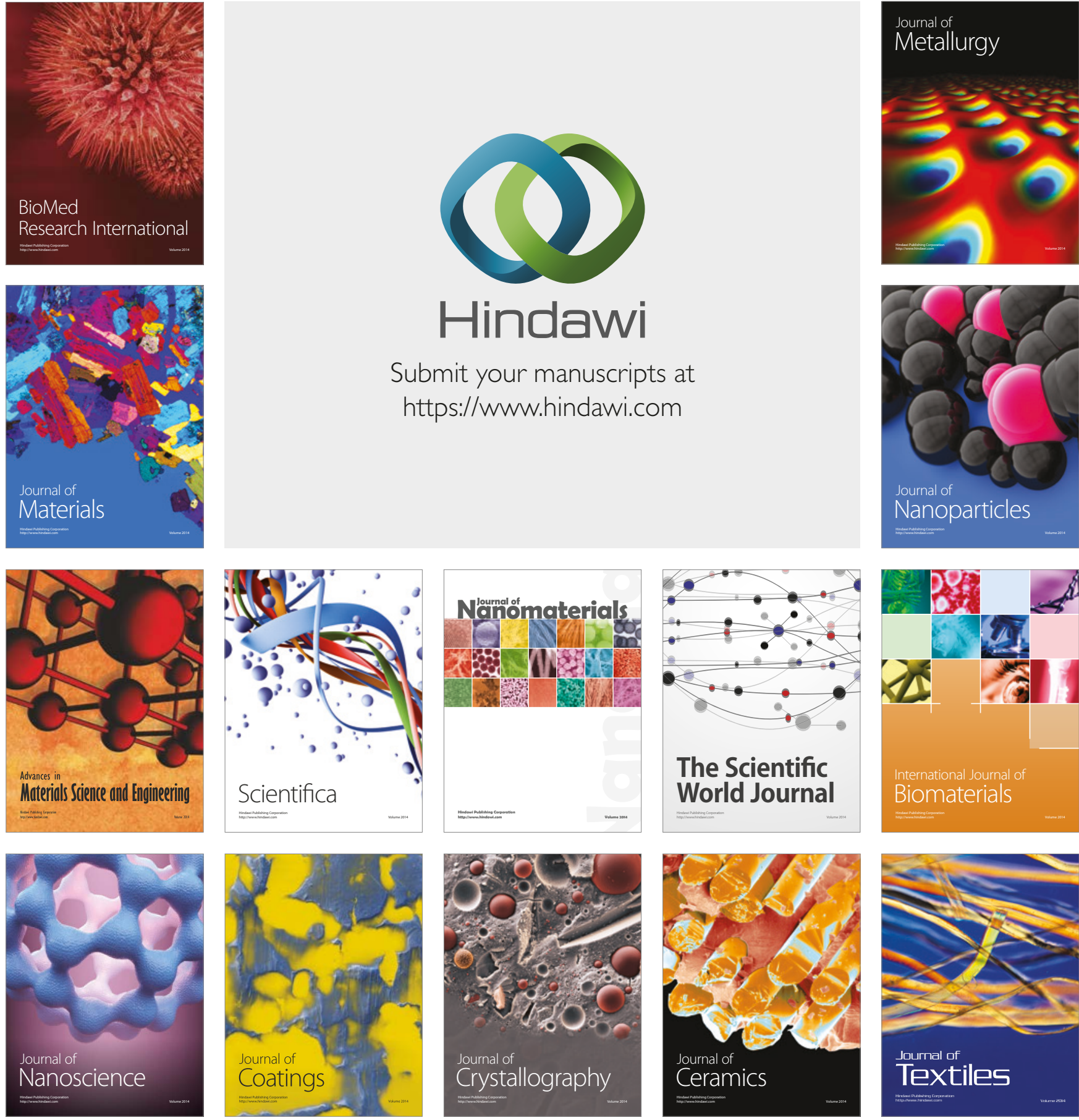

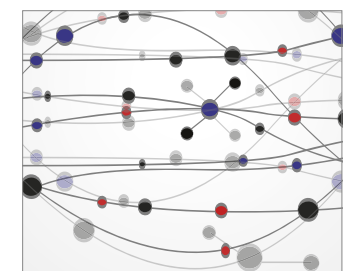

The Scientific World Journal
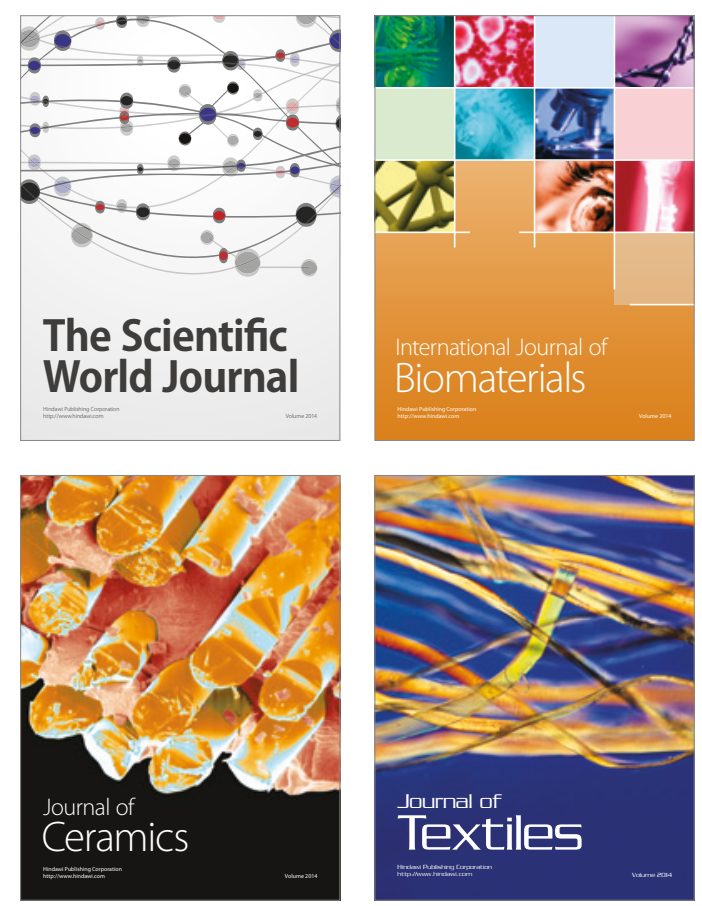